\title{
Self-Limiting Electrospray Deposition for the Surface Modification of Additively Manufactured Parts
}

\author{
Dylan A. Kovacevich ${ }^{\ddagger}, 1$, Lin Lei ${ }^{\ddagger}, 1$, Daehoon Han ${ }^{1}$, Christianna Kutznetsova1 ${ }^{1}$, Howon Lee ${ }^{1}$, Jonathan \\ P. Singer ${ }^{*} 1$
}

${ }^{1}$ Department of Mechanical and Aerospace Engineering, Rutgers University, New Jersey, 08854, USA

KEYWORDS: Electrospray deposition, additive manufacturing, functional coatings, polymers, self-assembly

\begin{abstract}
Electrospray deposition (ESD) is a spray coating process that utilizes a high voltage to atomize a flowing solution into charged microdroplets. These self-repulsive droplets evaporate as they travel to a target substrate, depositing the solution solids. Our previous research investigated the conditions necessary to minimize charge dissipation and deposit a thickness-limited film that grows in area over time through self-limiting electrospray deposition (SLED). Such sprays possess the ability to conformally coat complex three-dimensional objects without changing the location of the spray needle or orientation of the object. This makes them ideally suited for the post-processing of materials fabricated through additive manufacturing (AM), opening a paradigm of independent bulk and surface functionality. Having demonstrated three-dimensional coating with film thickness in the range of 1-50 $\mu \mathrm{m}$ on a variety of conductive objects, in this study we employed model substrates to quantitatively study the technique's limits with regard to geometry and scale. Specifically, we examined the effectiveness of thickness-limited ESD for coating recessed features with gaps ranging from $50 \mu \mathrm{m}$ to $1 \mathrm{~cm}$, as well as the ability to coat surfaces hidden from the line-of-sight of the spray needle. This was then extended to the coating of hydrogel structures printed by AM, demonstrating that coating could be conducted even into the body of the structures as a means to create hydrophobic surfaces without affecting the absorption-driven humidity response.
\end{abstract}

\section{INTRODUCTION}

Electrospray deposition (ESD) is a micro/nano-scale coating method for controllably depositing dilute solutions of monodispersed droplets via a high electric field. In the past few decades, this technology has been used with a range of applications. In biotechnology, ESD has been employed for the fabrication of protein microarrays and functional active proteins with high efficiency [1-3]. ESD has also been used for manufacturing photovoltaics and fuel cell systems, due to its advantages of less material loss and simple experimental setup $[4,5]$. Block copolymers deposited by ESD can produce thin films with well-ordered mesophases [6]. ESD can additionally be employed with suspended nanoparticles to functionalize surfaces with hierarchical nanoparticle assemblies [7-9] or as a means to seed cells [10].

In ESD, a voltage is applied to a solution flowing through a conductive nozzle, resulting in a fine spray of charged droplets. The most frequently used mode of spray, steady cone-jet, is characterized by the maintenance of a stable Taylor cone at the outlet of the nozzle through the balance of electrostatic forces and the surface tension of the solution [11]. At the tip of the cone, a microjet ejects a plume of drop- lets when a critical charge density is reached. These droplets undergo Coulombic fission to decrease the ratio of charge to surface area [12], resulting in a monodisperse spray of microdroplets.

The diameter of an electrosprayed droplet is determined by the properties of the solvent and the flow rate [13]:

$$
d=\alpha\left(\frac{Q^{3} \varepsilon_{0} \rho}{\pi^{4} \sigma \gamma}\right)^{\frac{1}{6}}+d_{o}
$$

Where $\alpha$ is a constant which related to the fluid's dielectric permittivity, $\rho$ is the density, $\gamma$ is the surface tension, $\sigma$ is the electrical conductivity, $Q$ is the flow rate, $\varepsilon_{0}$ is the permittivity of free space and $d_{o}$ is a small droplet diameter only significant at low flow rates. The maximum charge that can be carried by a droplet of diameter $d$ is approximated by the Rayleigh limit:

$$
q_{\max }=\pi\left(8 \varepsilon_{0} \gamma d^{3}\right)^{\frac{1}{2}}
$$

Taken together, these relations indicate that the delivered-charge per volume of spray can be expected to reduce with flow rate as $Q^{-\frac{1}{4}}$.

In a multitude of past results, it has been reported that ESD of dilute dielectric materials onto conductive surfaces can be used to deposit thin films. The morphologies of these films can be adjusted between dense, porous, or cracked by 
their spray conditions $[14,15]$. The key parameters are the substrate temperature, flow rate, volatility of the solvent, and the hydrophobicity of the spray materials. If the temperature is above the glass transition, $T_{g}$, of the solids or there is an excess of solvent or solvent vapor, then the ability of the film to redistribute charge by mass transport increases, leading to either viscous cellular instabilities or electrowetting into smooth films [16]. More recently, selflimiting effects have been reported, wherein charge buildup due to lack of material or charge mobility leads to a large reduction in the growth rate of the sprayed film thickness. Such a self-limiting effect has previously been observed in ESD $[17,18]$, but more extensively studied in electrostatic powder sprays. In this process, a thickness limit arises when Coulombic repulsion from the surface charge of the powder layer exceeds the attraction of image charges and van der Waals interactions [19]. However, electrostatic powder sprays differ from ESD in both scale and mechanism of the deposition. Electrostatic sprays usually involve the corona charging of powder with diameter in the range of 10-100 $\mu \mathrm{m}$ at a voltage of $40-100 \mathrm{kV}$ and are driven by pressurized air, rather than carried by a solvent $[20,21]$. Surface areas of several square meters are covered in minutes through this process, while ESD from a single Taylor cone can take an hour to cover the same area. A self-limiting effect is seen as undesirable in most electrostatic sprays, as the field that develops within the powder layer can cause a back-corona that dislodges material and damages the coating [21]. In contrast, ESD occurs at less-extreme conditions, allowing self-limiting to be employed with minimal risk of damaging the film.

Our recent work has characterized the criteria to achieve this thickness-limited self-limiting electrospray deposition (SLED) regime (Figure 1) [16]. We demonstrated that SLED will occur when spraying hydrophobic materials from a sufficiently volatile solvent, with a substrate temperature below the solid's glass transition temperature, $T_{g}$. For a given temperature, these sprays in a system of polystyrene (PS) in 2-butanone were observed to deliver repeatable coating thicknesses within a certain range of flow rates $(0.2 \mathrm{~mL} / \mathrm{hr}$ $\sim 1.5 \mathrm{~mL} / \mathrm{hr}$ ) and solids loadings (0.25 wt $\% 1.0 \mathrm{wt} \%)$ as a function of total sprayed solids mass. At the low end of both flow rate and concentration, solid conduction and water vapor in the ambient air provide paths for charge to leave the film, leading to thicker films. Within the SLED regime, the spray was shown to be capable of the three-dimensional coating of conducting objects with film thickness $<50 \mu \mathrm{m}$. Coverage of detailed surfaces and areas hidden from the line-of-sight of the needle was achieved on objects with overall dimensions on the scale of a few centimeters. The benefits of ESD in general as a three-dimensional coating process with regards to micro/nanoscale applications are (1) minimal material waste, (2) range of usable materials, (3) ability to operate in ambient conditions, and (4) precise control through the manipulation of electric fields and insulative patterning $[22,23]$. While, SLED places some limitations on the materials employed, there are additional opportunities in terms of guiding of the coating.
For SLED, the effects of the experimental parameters on the coating thickness all link to the strength of the driving electric field and the redistribution of charges on the coated surface, diagrammed in Figure 1a. In past ESD studies, shaped electric fields have been used to focus or manipulate the depositions. Lee et al used selective biasing of target electrodes to deposit multicolor LED pixels in mm-scale proximity [24]. Al-Milaji et al created hierarchical roughness by using a focusing biased conductive mask underneath the spray needle to create high aspect ratio structures, which were then resprayed without the mask and fluorinated to create super oleophobic materials [25]. Yan et al employed biasing on masks within the plane of the spray to control pattern sizes in a gradient, showing the control of electric field could be utilized for micro-manufacturing in electrospray deposition [23].

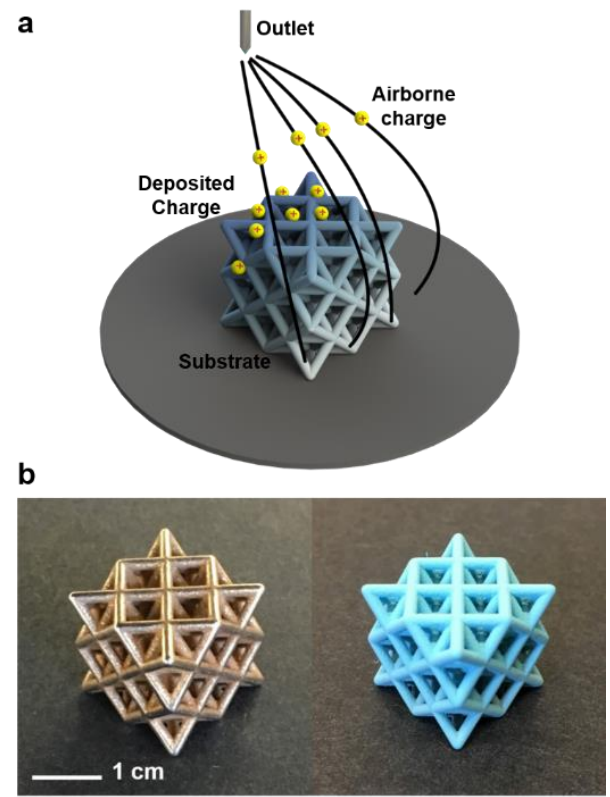

Figure 1. (a) Schematic of 3D coating with the SLED process where built up charge redirects the spray. (b) A steel-bronze 3D lattice before (left) and after (right) coating with blue dyed polystyrene:Kraton 1:1.

The electric field, not only as a function of applied voltages and spray height, but also the geometry of the target, will contribute to the final morphology. For electrostatic powder sprays, it is well-known that the Faraday cage effect causes a significant reduction in coverage for recessed areas [26]; high field strength at the recess entrance causes a buildup of charged material that prevents coating the interior. Less studied in ESD is the effects of curved or shadowed targets. Li et al examined ESD of fibers with conductive coatings of nanotubes [27]. Here, the local electric field, especially on the surface of fiber, influenced the uniformity of the coating with larger fibers possessing less-uniform coatings. Altman et al and our own work demonstrated that the creation of a repulsive field would mitigate this effect $[16,18]$. This is a similar effect to the repulsive field formed by an insulated stencil mask that has been shown to focus sprayed particles to form patterns at different sizes by Kim et al [28]. Our results on SLED-sprayed wires demonstrated that the final coating thickness on all surfaces depended 

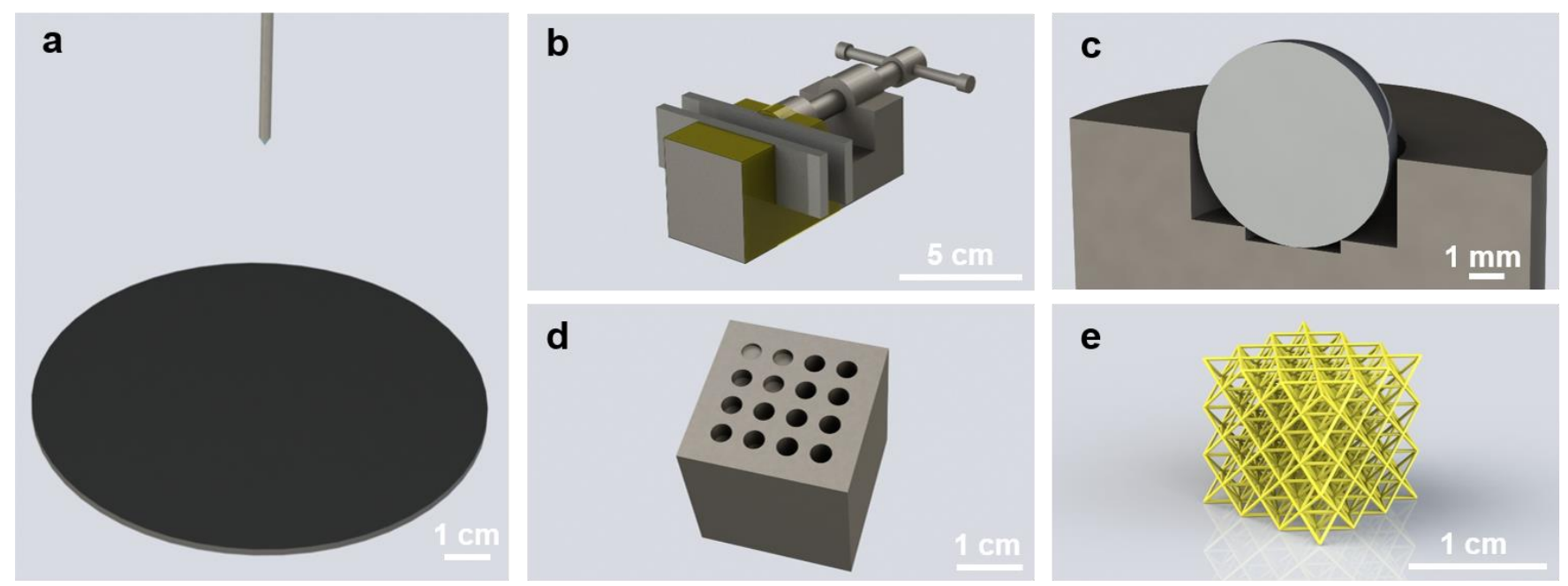

Figure 2. Model geometries: (a) 2D silicon wafer. (b) Uniform gap vise. (c) Pinch gap bearing. (d) Depth array holes. (e) AM hydrogel lattice.

only on the distance to the spray needle (i.e. the field strength) [16]. This trend was demonstrated on more complex 3D statues, but the effects of recessed or reentrant regions were difficult to analyze in aesthetically-selected sculpted morphologies. It was clear, however, that the conformal nature of the spray tracked these features within the statues, not filling or bridging recesses as might occur with fluid sprays. For this reason, there is an opportunity to apply SLED to structures produced through additive manufacturing (AM) as a complementary postprocessing method, demonstrated in Figure $1 b$.

Conformal coatings have been applied to AM structures previously to enhance various properties. Greer and colleagues have utilized atomic layer deposition (ALD) and electro(less)plating to create hollow ceramic and metallic lattices on removal of the polymer that demonstrated superior specific mechanical properties and enhanced toughness [29-31]. Key to many of these demonstrations is a decoupling of the surface and bulk properties of the material-the AM process is employed to define the scaffold of the structure while the post-processing either modifies the response of the superstructure to an external stimulus, or, as in the case of the hollow lattices, defines the entire properties of the final fabricated structure. The advantages presented by ESD, and specifically SLED, over these other techniques are: (1) the scalability and affordability of the process; (2) the ability to incorporate blends of materials such as active particles; and (3) the ability to hierarchically structure the final material. In terms of hierarchy, this can be implemented both by the field strength/distribution on a macro/microscale and also through the porous nature of the coatings on a micro/nanoscopic scale. The potential disadvantages of SLED arise from the non-uniformity of the coating thicknesses for certain applications that occur due to the field distribution or due to any geometric limitations with regards to minimum feature sizes. The significance of this effect would vary with application. For example, as an anticorrosion barrier, a certain minimum level of coverage may be sufficient, but as a mechanical actuator, the specific thickness map will determine the final deformed shape.

In this work, we examine the effects of the target geometry on the SLED coating thickness. Rather than begin with AM components, we first develop a better understanding of the relationship between the final film dimensions and electric field in gaps, holes, and bends by using model geometries of highly-conductive materials. We then apply these coatings to 3D hydrogel structures printed by AM [32, 33]. These hydrogel structures made of poly(ethylene glycol) diacrylate (PEGDA) and poly(acrylic acid) (PAA) have been demonstrated as stimuli-responsive underwater soft robotic applications and tunable mechanical metamaterials [32-34]. Changing the surface structure and hydrophobicity in turn will change how the material interacts with water or water vapor and may additionally limit fouling or unwanted ingress or egress of particulates. Another important feature from the standpoint of SLED is that hydrogel is an electrically insulating material that would normally be incompatible with ESD. By preswelling the structure with saline solution, we can bring it into the conductivity range where it is compatible with ESD in a similar fashion to the compatibility of electrostatic spray with biological systems in crop treatment. This is a potential route to spatially-controlled sprays in the future and also provides a means to prestress the external coating to allow for greater deformation of the underlying lattice.

For all of the sprays we employed PS with and without Kraton styrene-butadiene-styrene triblock copolymer as a plasticizer in 2-butanone at a concentration of $1 \mathrm{wt} \%$. Selflimiting sprays were conducted at room temperature, while sprays at $140^{\circ} \mathrm{C}$, above the glass transition of PS $\left(\sim 100^{\circ} \mathrm{C}\right)$, were performed to show contrasting behavior; this temperature was found to be in the charged-melt regime [16], which allows for the least material redistribution on arrival. As a baseline of coating a 2D surface, sprays at different voltages and needle heights were tested on a silicon wafer (Figure 2a), in order to fundamentally describe the relation- 
a

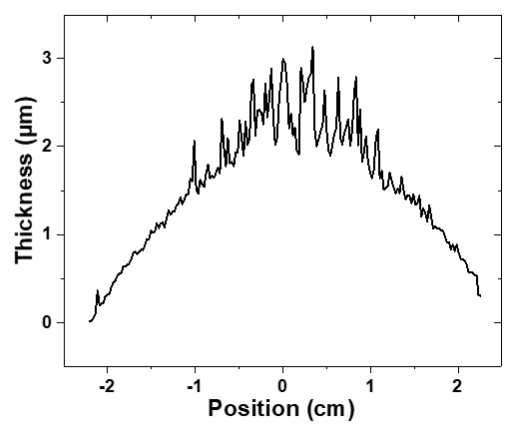

b

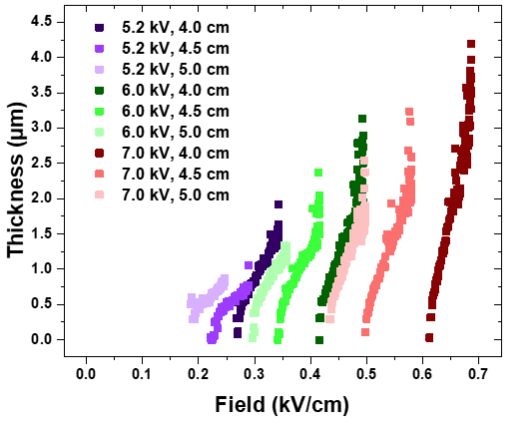

C

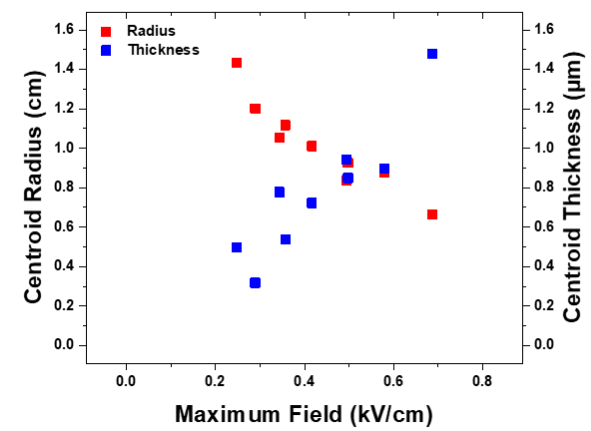

Figure 3. 1 wt.\% PS in 2-butanone sprayed at $0.5 \mathrm{~mL} / \mathrm{hr}$ for $30 \mathrm{~min}$ at $35^{\circ} \mathrm{C}$ on a silicon wafer: (a) Standard thickness-limited profile at $5.2 \mathrm{kV}$ and $4 \mathrm{~cm}$ needle height. (b) Thickness vs. initial effective electric field of sprays at varying voltages and heights. (c) Centroid coordinates of a half-profile dependent on the initial maximum electric field.

ship between the electric field and sprayed thin film thickness. To investigate the geometric effect and also for assessing the Faraday cage effect, we employed four kinds of characteristic geometries patterned from conductive materials-(1) a parallel plate gap with adjustable plate spacing (Figure 2b); (2) a shadowed curved cavity with a narrow entrance of variable size (Figure 2c); (3) a hole array of varying depth and constant diameter (Figure 2d); and (4) an hydrogel array of through holes of varying diameter (Figure S1). The specific aims of each geometry were figuring out how the dimensions of a thickness-limited coating changes related with sprayed geometries and electric field strength, then optimizing the self-limited coating on 3D objects in terms of controlling the film thickness and uniformity. All substrates were grounded during the spray period and the spray needle was stationary. After each spray trial, the resultant film was thermally smoothed to allow for thickness measurement by reflectometry, and a one-dimensional thickness profile was taken using an optical reflectometer microscope with a motorized stage. After studies on model geometries, compatibility with the hydrogel structures were confirmed using a hole array, and swelling and water interaction was demonstrated in a coated printed lattice (Figure 2e) with and without smoothing.

\section{RESULTS and DISCUSSION}

\subsection{Two-Dimensional Wafer}

Sprays onto a silicon wafer were performed at varying needle heights and voltages (Figure 2a).

Figure 3a shows a typical thickness profile of a SLED film, with optical reflectometry measurements taken at uniformly spaced points along a diameter of the coated region. The radial symmetry of the setup allows for the profile to be characteristic of the entire film area. Roughness in the center is likely due to the presence of solvent in the film. Large droplets result from brief periods of spray instability and do not fully evaporate prior to reaching the film. In Figure 3b, a series of film profiles at varying voltages and spray heights is plotted by initial effective electric field instead of position. The field was calculated through a two-dimensional axisymmetric COMSOL model (details in Supporting Information). The radius and thickness coordinates of the centroid are the center of the area formed by the intersection of half a thickness profile with the axes (calculation details can be found in the Supporting Information). These are plotted in Figure $3 c$ as a function of the maximum field. For the range of voltages and heights examined, the centroid radius decreases linearly with maximum field. The centroid thickness increases linearly with the maximum field.

The two-dimensional wafer sprays provide a baseline for understanding the relationship between the electric field and particle distribution for a thickness-limited spray. Calculation of the total mass in each film through the revolution of the spray profile shows that $\sim 95 \%$ of the sprayed mass was deposited on the substrate (calculation details can be found in the Supporting Information). There was no decrease in mass deposition efficiency for lower electric field strengths for the range of voltages and heights that were examined. Comparing the films by their respective maximum field strengths shows that a higher field focuses the spray in a generally linear fashion with regard to both thickness and radius. Changing the spray height affects both the maximum field strength as well as field profile shape, leading to some scattering in the trend. For each film, the thickness decreases linearly with the electric field corresponding to radial position. For films of higher maximum field, the thickness declines more steeply. This leads to the conclusion that a low field spray is more suitable for applications in which the uniformity of the coating over a large area is important.

\subsection{Uniform Gap Vise}

To investigate how the Faraday Cage effect influences coating thickness relative to depth, we started with a uniform gap model. Two parallel stainless steel plates were attached to a vise, with the capability to easily adjust spacing (Figure $2 \mathrm{~b})$. The spray needle was centered above the unmeasured plate at a height of $4 \mathrm{~cm}$. The gap width was varied from $50 \pm 18 \mu \mathrm{m}-1 \pm 0.03 \mathrm{~cm}$.

For each trial, 1D film thickness measurements were taken down the depth of one of the plates and plotted in Figure 4a. In each of the depth vs. film thickness plots, two apparently linear regimes were present. A steep decrease in thickness occurred near the top of the plate, while a more gradual decline occurred near the bottom. Thicknesses at 
a

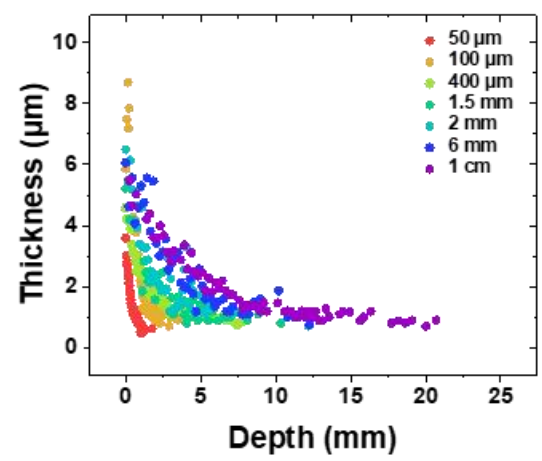

c i

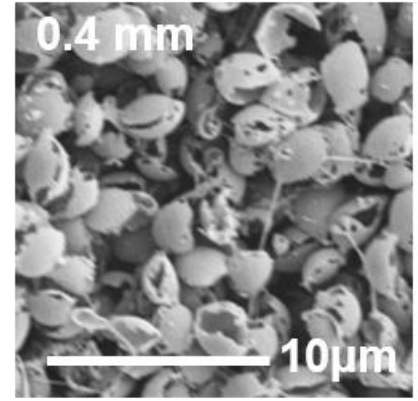

b

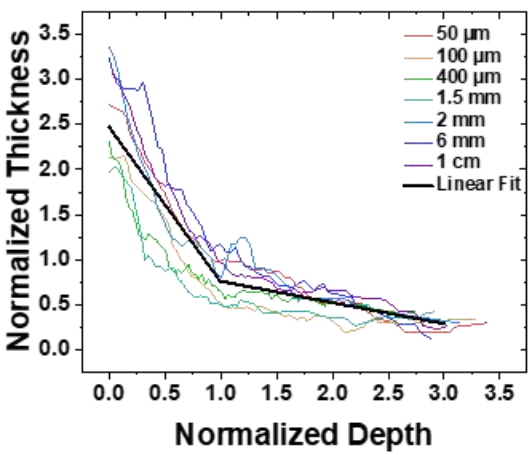

iii d

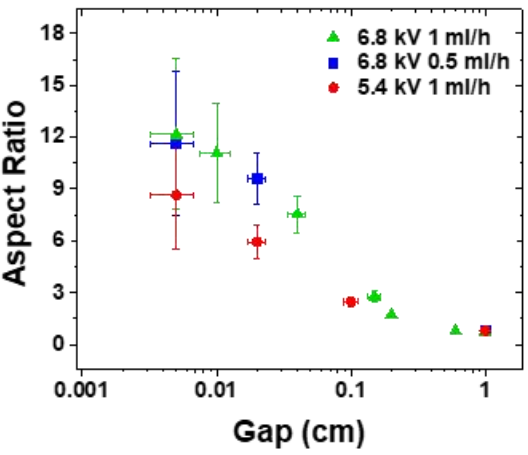

iv
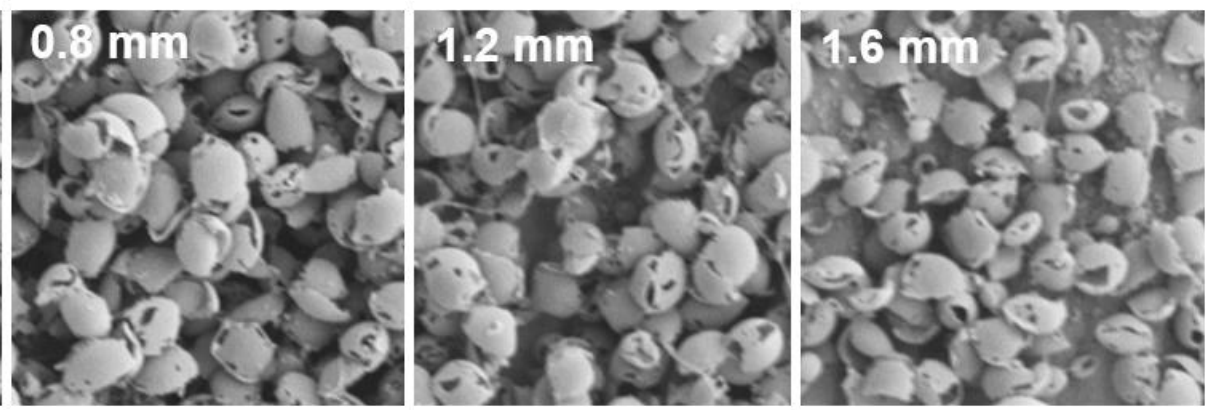

Figure 4. Sprays of $1 \mathrm{wt} . \%$ PS at $1 \mathrm{~mL} / \mathrm{h}$ for 1 hour at room temperature: (a) Thickness vs. depth down the plate for $6.8 \mathrm{kV}$ and gap sizes ranging from $50 \mu \mathrm{m}$ to $1 \mathrm{~cm}$. (b) Thickness vs. depth both normalized by the coordinates of their respective centroids, which is typically located at a third of the maximum thickness and maximum length. (c) SEM images down the vise plate in $0.4 \mathrm{~mm}$ increments for a $6.8 \mathrm{kV} 1 \mathrm{ml} / \mathrm{h}$ spray on a $50 \mu \mathrm{m}$ gap. (d) Ratio of centroid depth/gap vs. gap.

the top of the plate are similar across gap dimension, with some variation attributed to inconsistency in the initial measurement position (centering and distance from the top), When the results at all gap sizes were plotted with normalized depth and thickness using the centroid coordinates, it is evident that the regimes occur at the same ratio of depth down the plate, as shown in Figure 4b. In Figure 4c, the ratio of the centroid depth to gap size is plotted as a function of the gap size, with additional sprays at $5.4 \mathrm{kV}$ and $0.5 \mathrm{ml} / \mathrm{h}$. Figure $4 \mathrm{~d}$ shows SEM images taken at $0.4,0.8,1.2$, and 1.6 $\mathrm{mm}$ down the plate, with the coating showing the characteristic polymer shell morphology characteristic of SLED sprays [16]. The greatest depth plotted in Figure 4a is 1.76 $\mathrm{mm}$, so the first image falls in the steep section of the thickness profile, while the remaining three are in the flatter one.

Thickness profiles down the length of a plate are characterized by two phases. The upper third of the coated length has a steep decline in thickness, while the remaining two thirds of the profile is comparatively shallow. The centroid depth and thickness are located near the transition between these phases. The steep initial section is the result of high electric field at the gap entrance, described as the Faraday effect. The origin of the latter section can be understood by viewing ESD in the frame of a time dependent process. The initial spray area receives a relatively constant deposition of particles until the film thickness nears the limiting value. At this point, the film gains surface charge that no longer can be dissipated through the grounded substrate and incoming charged particles are redirected. The charged-gap entrance repels most particles, but can also act as a lens that focuses them down the length of the plate. This results in the long thin section of the profile. These two profile sections are increasingly distinct as the gap sizes decreases due to the greater field gradient. At larger gaps, the thickness profile more closely approximates a $1 / \mathrm{r}$ curve. The centroid being located at the transition is a feature of the symmetry of the reciprocal function. Figure $4 \mathrm{c}$ shows that the size of the PS shells is constant down the length of the plate, removing the possibility that the second phase arises from variance in the sprayed droplets, such as satellite particles. Additionally, images taken in the second phase (Figure $4 \mathrm{~d}$ rightmost three) show that the majority of the length has a full layer of shells, so the flatness of the thickness profile is not an artifact of particle clustering and plot smoothing. When determining if an object with a recessed feature will receive suitable coverage, it is important to consider the aspect ratio of the feature, which we define as the depth of the centroid divided by the gap width (Figure $4 d$ ). At larger gap sizes, the depth of coating is roughly the size of the gap, with the ratio increasing as the gap size decreases. Lower spray voltage resulted in less coating depth as well as a thinner film. This may be attributed to the tendency of a lower voltage film to have a wider area of coverage as seen in the two-dimensional sprays. Because the vise gap is directly underneath the spray needle, a high voltage is more effective at focusing particles downwards. A lower flow rate was used to examine if the droplet diameter, and resultant shell size, has an effect on the spray coverage. According to Eq. 1, the droplet 
diameter is proportional to the square root of the flow rate. The influence of particle size should be expected to increase as the gap decreases, where the minimum gap is limited by the diameter of a single shell. However, there was no significant change in depth of coverage in the range of $50 \mu \mathrm{m}$ to 1 $\mathrm{cm}$. Considering the implication of the results, we observed aspect ratios of up to 12 , with an s-curve like behavior beginning to emerge at the lowest gaps suggesting that there would be some limit to the depth achievable for these specific parameters, though longer times and higher fields may have pushed this value. This means that significant amounts of coating were penetrating $20 x$ the gap size for the smallest gaps, up to $1 \mathrm{~mm}$ for the $50 \mu \mathrm{m}$ gap, demonstrating SLED to be more effective in micro-manufacturing processes.

\subsection{Pinch Gap/Cavity Bearing}

To study the capability of coating a cavity, a ball bearing was placed in a cylindrical recess in a steel block. This formed an annular region that widens with depth and has surfaces hidden from the spray needle's line-of-sight (Figure 2c). After the spray period and thermal smoothing, a one-dimensional thickness profile was taken along a radius of the surface recessed $2.75 \mathrm{~mm}$ into the block.
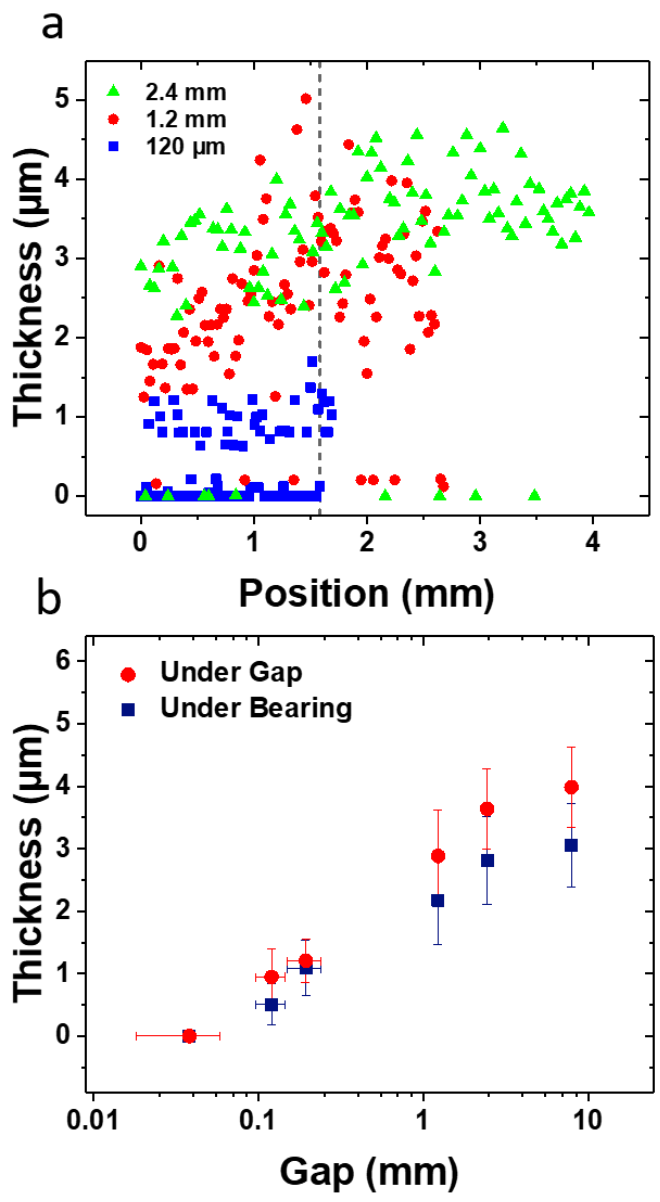

Figure 5. Sprays of $1 \mathrm{wt} . \%$ PS for 1 hour at room temperature and a height of $4 \mathrm{~cm}$ : (a) Thickness vs. radial position (outward from the contact point of the bearing and recessed surface) profiles for varying gaps, with data to the left of the dashed line in the area shadowed by the bearing. (b) Mean thickness vs. gap size, separated by region under the bearing and under the gap.

Figure 5a shows how the thickness of the film varies with radial position relative to the bearing, where zero indicates the point at which the recessed surface contacts the bearing underside. The dashed line separates the substrate area hidden from the line-of-sight of the spray needle and that directly under the gap. The notable number of sampled points in the $120 \mu \mathrm{m}$ profile that appear to have no coating could be due to particles clustering instead of forming a full layer or a result of dewetting during the thermal smoothing process in very thin films. Figure $5 \mathrm{~b}$ shows how the average thickness of the film varies with gap sizes for these two regions.

The single bearing cavity model combines the variable gap of the vise model with interior geometry hidden from the line-of-sight of the spray needle. Additionally, the depth the film is measured at is constant and the substrate surface perpendicular to the spray needle. The thickness of the film does decrease gradually in the region underneath the bearing, but there is not a significant buildup in coating material directly below the gap. This indicates that electrosprayed particles are capable of changing trajectory near the charged film surface to reach ground even after being focused through a narrow area. The gradual decline in thickness in the section under the bearing could be due to a combination of reduced field with distance as well as the decreased space between the underside of the bearing, which also receives a charged coating, and the flat surface. The average thickness of coating varies logarithmically with gap size, as shown in Figure 5c. At a near centimeter gap, the gain in film thickness flattens as the Faraday effect becomes less significant. It should be expected that eventually the thickness decreases at even larger gaps, where the distance of the substrate from the needle plays a larger role in reducing the electric field than the proximity of the charged gap surfaces. The smallest tested gap of $38 \mu \mathrm{m}$ received no coverage, which is unsurprising since the depth of the cavity is $2.75 \mathrm{~mm}$, translating to an aspect ratio of $\sim 36$ with respect to the gap to the limit of coating. Indeed, that there was a considerable coating at the $120 \mu \mathrm{m}$ gap, indicates that this geometry was more favorable for the spray penetration than the flat vice. This is possibly due to the widening of the gap under the lensing aperture providing less screening of the gap as spray built up within the cavity. It is also instructive to consider how rapidly course changes are occurring in the droplets. While the film is highly influencing the electric fields the droplets experience, if we approximate the droplet velocity as the terminal velocity experienced in a usual ESD experiment, the droplets would have to turn at an angular velocity of $>500 \mathrm{rad} / \mathrm{s}$ in the case of the $120 \mu \mathrm{m}$ gap to reach the limits of the cavity. This indicates that the droplets are capable of dealing with rapid changes in topography, such as highly-tortuous paths.

\subsection{Depth Array}

An array of holes of constant diameter and series of depths in a stainless steel block was used to test how the coating thickness varied with depth in a single trial(Figure 
2d). This allowed for quick comparison of the effects of temperature (charge mobility), applied voltage, and spray time. The holes had a diameter of $0.32 \mathrm{~cm}$ and depth ranging from $0.05 \mathrm{~cm}$ to $0.81 \mathrm{~cm}$. Three thickness measurements were taken near the center of the recessed surface of each hole and averaged.
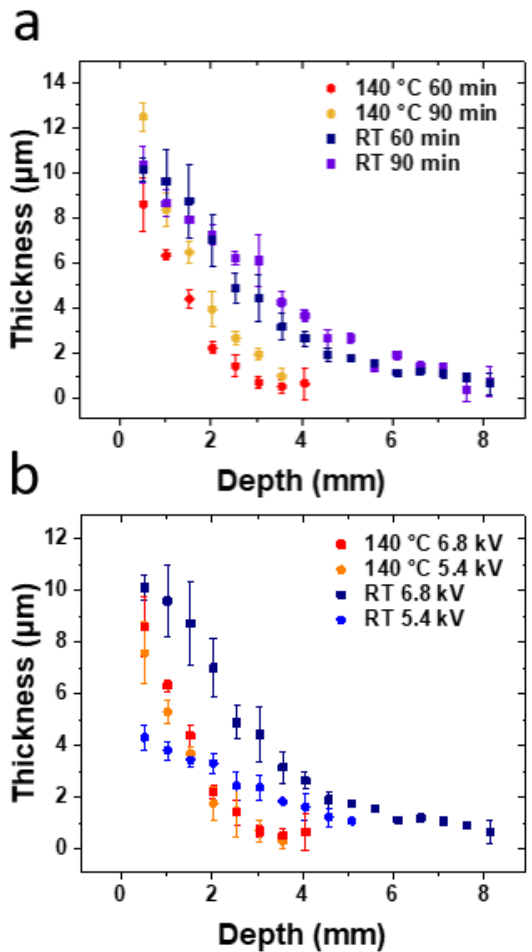

Figure 6. Thickness vs. depth of hole plots for sprays of 1 wt.\% PS at a flow rate of $1 \mathrm{ml} / \mathrm{h}$ and a height of $4 \mathrm{~cm}$ : (a) At 6.8 $\mathrm{kV}$ for a spray period of 60 or $90 \mathrm{~min}$ and at room temperature or $140^{\circ} \mathrm{C}$. (b) At 5.4 or $6.8 \mathrm{kV}$ for a period of $60 \mathrm{~min}$ and at room temperature or $140{ }^{\circ} \mathrm{C}$.

Figure 6 a compares the thickness profiles of room temperature and $140{ }^{\circ} \mathrm{C}$ sprays at 60 and $90 \mathrm{~min}$, with a voltage of $6.8 \mathrm{kV}$. The overlap of the room temperature depth vs. thickness curves at $60 \mathrm{~min}$ and $90 \mathrm{~min}$ indicates that the spray is thickness-limited by $60 \mathrm{~min}$. In contrast, the $140^{\circ} \mathrm{C}$ $90 \mathrm{~min}$ profile gains thickness proportional to its $60 \mathrm{~min}$ thickness at each depth. The room temperature spray profiles display a similar trend to the vise sprays, with a steep decline in thickness followed by a more uniform region. The $140{ }^{\circ} \mathrm{C}$ profiles do not feature a secondary region. In Figure $6 \mathrm{~b}$, the two temperature profiles are compared at $5.4 \mathrm{kV}$ and $6.8 \mathrm{kV}$. At the lower voltage, the primary region is less steep, and the flatter region is not visible. The different voltage curves at $140{ }^{\circ} \mathrm{C}$ overlap.

The depth array trials were performed at both room temperature and $140{ }^{\circ} \mathrm{C}$ to contrast the spray behavior of a thickness-limited film with a mobile-charge film. At $140{ }^{\circ} \mathrm{C}$, the rate at which the PS film dissipates charge increases significantly through both increased solvent swelling and mobility due to being over its glass transition temperature. The room temperature spray depth versus thickness curve is similar to the thickness profile of a vise plot. Both have an initial steep decline in thickness followed by a longer, thin tail. Only the first regime is visible in the high temperature plots. This supports the conclusion that the first regime is representative of the initial direct spray area, while the second regime is a result of repulsion from a film which cannot dissipate surface charge. Comparison of the 60 and $90 \mathrm{~min}$ trials for each temperature highlights the difference in thickness growth rates between a self-limited and mobilecharge film. The room temperature depth versus thickness curves for each period are coincident, as past $60 \mathrm{~min}$ the film has reached a thickness at each depth where charge can no longer dissipate. Incoming charged particles are repulsed from the upper cube surface and instead wrap around the sides. The high temperature 90 min curve displays an increase in thickness at each depth proportional to the thickness at $60 \mathrm{~min}$, suggesting a linear growth rate. Charge dissipates quickly enough that the initial electric field is minimally altered.

\subsection{D Lattice Structured Hydrogel}

After observing the effects of isolated features on the final electrosprayed coating, we verified that we could infiltrate holes as small as $150 \mu \mathrm{m}$ in a 3D-printed PAA hydrogel plate manufactured through projection micro-stereolithography $(\mathrm{P} \mu \mathrm{SL})$ [35-37] (see Supporting Information). This established, we moved to lattices to demonstrate the ability to coat a more complex structure as well as practical surface property modification (Figure 2e). These were $1 \mathrm{~cm}$ edge length cubes with an octet truss structure. This lattice was composed of tetrahedral skeletons with $2.5 \mathrm{~mm}$ beam length and $250 \mu \mathrm{m}$ beam diameter. To reach the grounded sections of the structure, the electrosprayed particles had to pass through gaps of $1.2 \mathrm{~mm}$ at $110^{\circ}$ angles. This geometry combines the narrow openings of the vice and cylindrical recess studies with the tight curves and shadowed surfaces of the bearing study. Notably, in Figure 3c., a coating at a vice gap size of $1.2 \mathrm{~mm}$ would have a centroid depth of $\sim 3 \mathrm{x}$ that, or a total depth of $\sim 1.1 \mathrm{~cm}$, slightly over the height of the hydrogel cube. This indicates that SLED should be capable of coating the entirety of the structure. While the lattice is more complicated than a uniform gap and the coating may be able to actually reach deeper due to additional void space, this analysis shows how the prior study may serve as a useful guideline.

A hydrogel lattice was sprayed with a 1:1 Kraton to PS 1 wt. \% solids loading solution and then sliced down the center. Several images of the cross section are shown in Figure 7. The lighter regions are the coating, while the darker regions are uncoated areas that were sliced across or damaged from handling. The ridges are the z-level byproduct of the stereolithography process. 


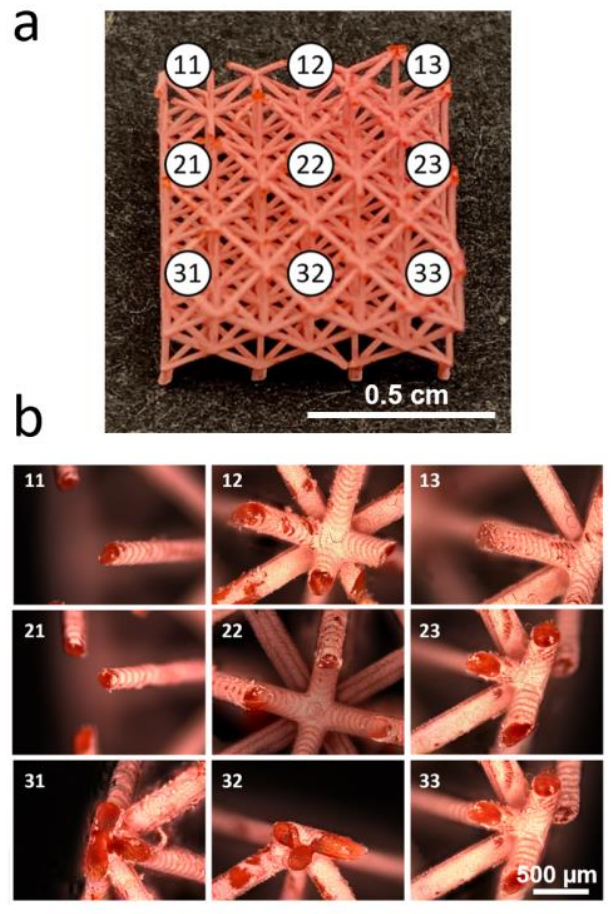

Figure 7. Coated hydrogel lattice sliced through the center in the xy plane: (a) Bottom half of the lattice labeled with the coordinates of corresponding (b) microscope images showing the light-colored coating and darker hydrogel.

A coating video is available as Supplementary Video 1. During coating, the water within the hydrogel was observed to rapidly dry, and certain high-crosslinked prints (as evidenced by low overall swelling) could not be fully coated while the gel remained hydrated. Figure 8a shows the microstructure of a single uncoated beam from the side of the lattice. The beam was aligned vertically in the image, but is at a $45^{\circ}$ on the lattice. This can be seen by the angled print layers, labeled as ridges on the coated beam in figure $8 \mathrm{~b}$. The coating conforms to the ridges rather than filling them, indicating SLED is capable of preserving detailed features. Upon swelling in ambient humidity, cracks in the powder form rather than a full delamination of the coating from the growth of the gel with humidity. These cracks, which are also visible in Figure $8 \mathrm{~b}$, allow the gel to be repeatedly swollen without the destruction of the coating. In Figure 8c, exposure to acetone vapors fused the individual coating particles to form a smooth film. The ridges of the original surface are still visible, but the coating also has a scaly appearance due to the precursor cracks in the powder.

a

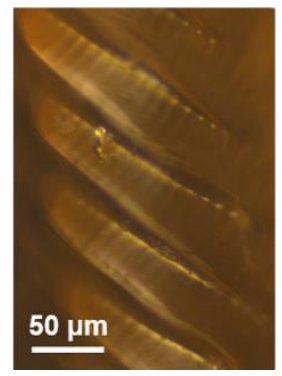

b

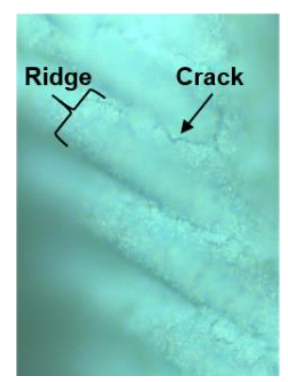

C

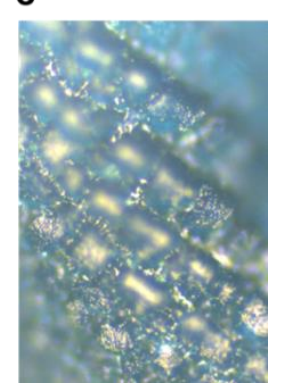

Figure 8. Microstructure of a lattice outer strut: (a) Uncoated. (b) Coated with PS, including plasticizer and dye. (c) Coated and smoothed with acetone vapors.

To assess the ability of water to diffuse into the hydrogels, a dry lattice was left in a controlled high humidity environment for a period of $4.5 \mathrm{hrs}$ and its swelling was monitored by camera. This was repeated for three trials with two lattices. The lattices were then coated in a 1:1 polystyrene and Kraton blend via SLED for $3 \mathrm{hrs}$ at $0.5 \mathrm{~mL} / \mathrm{hr}$. One lattice was sprayed at $5.4 \mathrm{kV}$ and the other at $6.8 \mathrm{kV}$, which was later smoothed via acetone vapors. The three swelling trials were then repeated and the results compared. A swelling video is available as Supplementary Video 2. Figure 7 shows the initial lattice and its swollen state after $30 \mathrm{~min}$ in high humidity. a

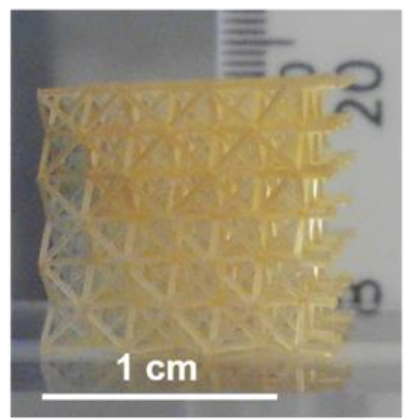

C

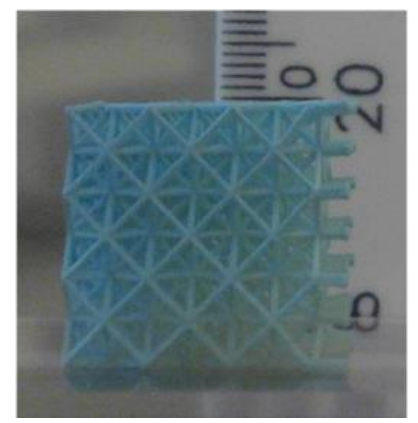

b

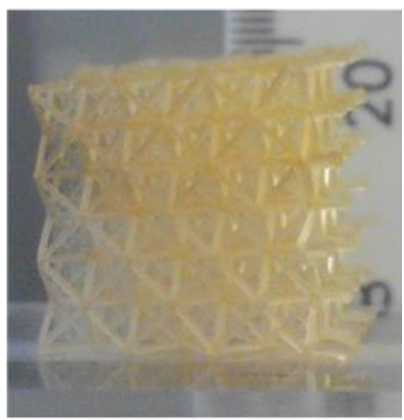

d

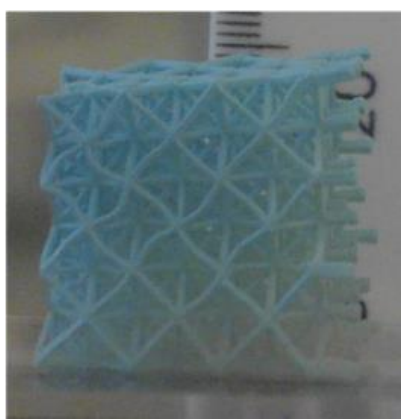

Figure 9. Lattice swelling in a high humidity environment for 30 min: (a-b) Uncoated. (c-d) Coated in a 1:1 PS Kraton blend at $5.4 \mathrm{kV}$ for $3 \mathrm{hrs}$ with spray needle $4 \mathrm{~cm}$ above the upper left edge.

Both the uncoated and coated lattices' overall dimensions expanded at similar rates as shown in Figure 9, indicating that the rate of moisture diffusion was unaffected by the coating. The hydrogels experienced a period of fast growth before they approached a limiting size. During this period, the coated lattice exhibited structural buckling which can be seen in Figure 9d. These changes occurred due to anisotropy in the lattice's mechanical strength, resulting from the gradient in coating thickness from the upper left to bottom right corner. The thicker-coated areas expanded at a slower rate than those thinly-coated, causing the beams to buckle and the overall lattice to distort. The buckling was most prevalent in the hydrogel coated at $5.4 \mathrm{kV}$, while still visible in the $6.4 \mathrm{kV}$ coated lattice. It occurred minimally in the uncoated lattices or the $6.4 \mathrm{kV}$ acetone vapor-smoothed lattice. This indicates that for bending to occur, the mechanical 
strength must be anisotropic and below a limiting value for some portion. A sufficiently thick or fused coating provides too much rigidity for the distortion of the lattice.

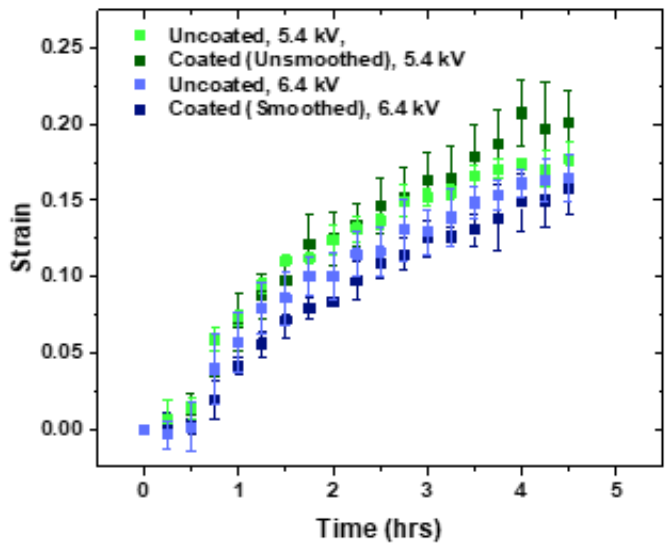

Figure 10. Growth rates for uncoated, coated, and smoothed coated lattice in a high humidity environment. Each series is the average of three cycles.

A coated unsmoothed lattice and an uncoated lattice were place in dishes of water to compare macroscale surface interactions, shown in Figure 9a. The most thickly coated face of the lattice rested on the water surface with minimal area of contact. When forced downward, the lattice quickly sprang back to the surface without water penetrating the interior (Supplementary Video 3). In contrast, the uncoated lattice was flooded and remained partially submerged (Supplementary Video 4). The water-repelling behavior of the coated lattice can be explained by the hydrophobicity of the coating material as well as the hierarchical shell microstructure shown earlier in Figure 4d. The water contact angle of the same coating on a silicon wafer was found to be $132^{\circ}$ (see Supplementary Information). In Figure $11 \mathrm{~b}$ it can be seen that the thinly coated underside beams of the lattice distorted after the full lattice was submerged due to the anisotropy in swelling rates.

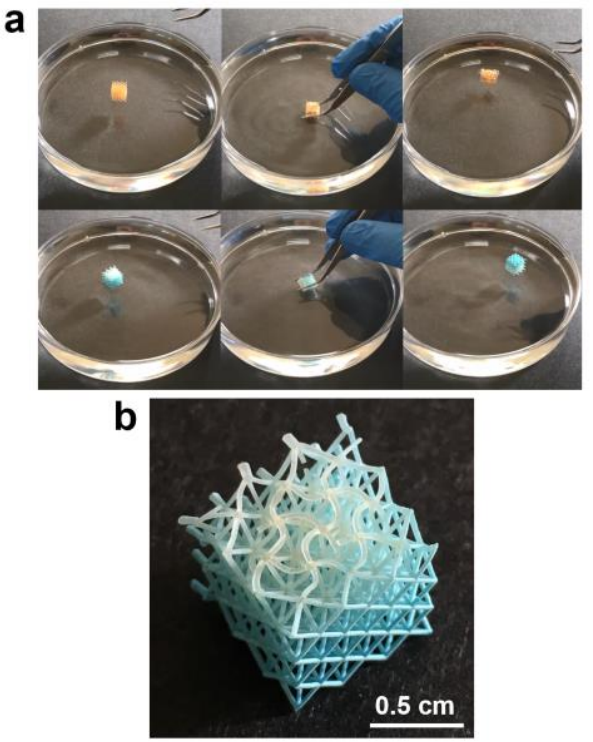

Figure 11. Lattice interaction with water: (a) Uncoated (top) and coated (bottom) lattices in a water-filled dish before, during, and after submersion. (b) Lattice with bent beams on the minimally-coated underside after submersion.

\section{CONCLUSIONS}

The three-dimensional coating capability of ESD when using an insulator as a charge carrier adds to its already versatile range of applications in depositing minute amounts of material. This process may provide an efficient and inexpensive method for conformally coating structures with features on the micro-scale under ambient conditions.

Through the use of model substrates, we have improved our understanding of how the dimensions of a SLED coating vary with substrate geometry and electric field strength. This enables improved determination of an object's suitability for coating as well as more precise control over the thickness and uniformity of the final film. For recessed features, we have identified two stages of self-limited spray: (1) the initial spray region near the entrance receives a thick coating layer until a strong counter-field is established, which then, through a focusing effect, results in (2) an increase in film depth that is characterized by a relatively thin and flat thickness profile. The second stage of spray is effective at coating the interior of cavities with large surface area hidden from the line-of-sight of the spray needle.

An application of ESD as a method for the post-processing of additively manufactured structures was demonstrated by modifying the surface properties and mechanical strength of 3D lattice hydrogels printed via P?SL. A porous polymer coating allowed the hydrogels to retain their ability to swell in high humidity environments, while simultaneously preventing wetting on the macroscale. Additionally, gradients in the coating thickness caused anisotropy in the lattice rigidity and induced bending in the more rapidly expanding beams. Utilizing an external field or deliberate selection of geometry could allow for spatially tunable coating thickness and resultant properties.

\section{EXPERIMENTAL SECTION}

\subsection{Materials}

Polystyrene (molecular weight $(\mathrm{MW})=35 \mathrm{kDa}$, SigmaAldrich) and Kraton D1102 (Hexion) were used as received from the manufacturer. 2-Butanone ( $>99 \%$, Sigma-Aldrich) was used as received as the carrier solvent for the spray solutions. Coumarin 466 dye $(\lambda e x=380 \mathrm{~nm}, \lambda e m=460 \mathrm{~nm}$, LambdaPhysik) and Copper (II) 2,9,16,23-tetra-tert-butyl$29 \mathrm{H}, 31 \mathrm{H}$-pthalocyanine (97\%, Sigma-Aldrich) was used as received.

All chemicals for 3D printing of hydrogel structures were used as received. Poly(ethylene glycol) diacrylate (PEGDA, Mn 250) (Sigma-Aldrich) and acrylic acid (AA) (Sigma-Aldrich) were used as monomers. Bisphenol A ethoxylate dimethacrylate (BPA, Mn 1700) was used as a crosslinker for AA. Phenylbis(2,4,6-trimethylbenzoyl) phosphine oxide (Sigma-Aldrich) was used as a photo-initiator (PI). Sudan I (Sigma-Aldrich) was used as a photo-absorber (PA).

\subsection{Electrospray Setup}


The main components of the electrospray setup include a syringe pump (KD Scientific KDS-100) and two high-voltage potential supplies (Matsusada Precision Inc., RB30-30P) connected to a stainless steel needle (SAI Infusion, 20 gauge, $1.5^{\prime \prime}$ ) and a steel focusing ring. The ring had an inner diameter of $2 \mathrm{~cm}$ and an outer diameter of $4 \mathrm{~cm}$ and was held 1 $\mathrm{cm}$ above the needle outlet. Sprays were performed on a hot plate inside of a fume hood. Films deposited at a temperature below the $T_{g}$ were smoothed thermally before mapping with a microscopic reflectometer (Filmetrics F40), which was customized with a motorized stage (Zaber E13F33E) and manual control and mapping software.

\subsection{Substrate Setup}

Plane - A silicon wafer $10 \mathrm{~cm}$ in diameter was clipped with a ground wire to a heating plate.

Uniform Gap - A stainless steel plate $(8.5 \times 2.5 \times 0.5 \mathrm{~cm})$ was secured to each jaw of a vise (Palmgren) and a ground wire was clipped to the back of the vise. Kapton tape was used as an insulator to prevent coating of undesired surfaces.

Pinch Gap/Cavity - A stainless steel ball bearing $0.635 \mathrm{~cm}$ in diameter was placed in a cylindrical recess in the upper surface of a $2.5 \mathrm{~cm}$ stainless steel cube. During spray, this cube sat on a silicon wafer clipped with a ground wire to a heating plate.

Depth Array - A $2.5 \mathrm{~cm}$ stainless steel cube with 16 holes of diameter $0.3175 \mathrm{~cm}$ and depths ranging from $0.05-0.81$ $\mathrm{cm}$ sat on a silicon wafer clipped with a ground wire to a heating plate.

Hydrogel - The hole array hydrogel structure was 3D printed through $\mathrm{P} \mu \mathrm{SL}$ of a precursor solution including PEGDA as a monomer, 2 wt $\%$ PI, and $0.1 \mathrm{wt} \%$ PA. The structure consisted of 25 layers, each of which was $20 \mu \mathrm{m}$ in thickness. Each layer was photopolymerized with patterned UV light ( $405 \mathrm{~nm}, 10 \mathrm{~mW} \mathrm{~cm}$-2) for $1.5 \mathrm{~s}$. The final structure had overall dimensions $1.6 \mathrm{~mm} \times 1.6 \mathrm{~mm} \times 500 \mu \mathrm{m}$ and four rows of holes, each with diameter 150, 200, 250, $300 \mu \mathrm{m}$. The printed structure was then rinsed in ethanol for $5 \mathrm{~min}$ to remove uncured precursor solution.

3D Hydrogel Lattice- A precursor solution for the PAA hydrogel lattice structures was prepared by mixing AA as a monomer and BPA as a crosslinker at a ratio of 55:45 in weight. PI and PA were added into the mixed solution at the concentration of $2 \mathrm{wt} \%$ and $0.1 \mathrm{wt} \%$, respectively. The 3D lattice consisted of 198 layers, each of which was $50 \mu \mathrm{m}$ in thickness. Each layer was photopolymerized with patterned UV light ( $365 \mathrm{~nm}, 29 \mathrm{~mW} \mathrm{cm-2)} \mathrm{for} 5 \mathrm{~s}$. The final lattice structure had overall dimensions $1 \mathrm{~cm} \times 1 \mathrm{~cm} \times 1 \mathrm{~cm}$. After printing, the printed structures were rinsed 4 times with ethanol to remove uncured precursor solution.

\section{ASSOCIATED CONTENT}

Supporting Information. Figures and discussion of hydrogel hole-array, COMSOL FEM electrostatic simulation of the wafer fields, details of centroid and mass calculations, and a table of experimental parameters. Video of the hydrogel lattice coating process, swelling, and submersion testing.

\section{AUTHOR INFORMATION}

\section{Corresponding Author}

* Jonathan P. Singer, 98 Brett Road, Piscataway, NJ 08854

\section{Author Contributions}

The manuscript was written through contributions of all authors. All authors have given approval to the final version of the manuscript. $\$$ D.A.K. and L.L. contributed equally. (match statement to author names with a symbol)

\section{Funding Sources}

J.P.S. acknowledges the 3M Corporation through the 3M NonTenured Faculty Award. D.A.K. and C.K. acknowledge the New Jersey Space Grant Consortium, funded by NASA, through their student fellows program. H.L. acknowledges the Haythornthwaite Foundation Research Initiation Award. H.L. and D.H. acknowledge Rutgers University Research Council Grant.

\section{ABBREVIATIONS}

ESD, electrospray deposition; SLED, self-limiting electrospray deposition; AM, additive manufacturing; PS, polystyrene; ALD, atomic layer deposition; PEGDA, poly(ethylene glycol) diacrylate; PAA, poly(acrylic acid); SEM, scanning electron microscopy; $\mathrm{P} \mu \mathrm{SL}$, projection micro stereolithography; micro-CT, micro computed tomography; MW, molecular weight; BPA, bisphenol A ethoxylate; PI, photo-initiator; PA, photo-absorber.

\section{REFERENCES}

1. Morozov, V.N. and T.Y.J.A.c. Morozova, Electrospray deposition as a method to fabricate functionally active protein films. 1999. 71(7): p. 1415-1420.

2. Avseenko, N.V., et al., Immunoassay with multicomponent protein microarrays fabricated by electrospray deposition. 2002. 74(5): p. 927-933.

3. Morozov, V.N. and T.Y.J.A.c. Morozova, Electrospray deposition as a method for mass fabrication of mono-and multicomponent microarrays of biological and biologically active substances. 1999. 71(15): p. 3110-3117.

4. Zhao, X.-Y., et al., Enhancement of the performance of organic solar cells by electrospray deposition with optimal solvent system. Solar Energy Materials and Solar Cells, 2014. 121: p. 119125 .

5. Martin, S., P.L. Garcia-Ybarra, and J.L. Castillo, Electrospray deposition of catalyst layers with ultra-low Pt loadings for PEM fuel cells cathodes. Journal of Power Sources, 2010. 195(9): $\mathrm{p}$. 2443-2449.

$6 . \quad H u$, H., J.P. Singer, and C.O. Osuji, Morphology Development in Thin Films of a Lamellar Block Copolymer Deposited by Electrospray. Macromolecules, 2014. 47(16): p. 5703-5710.

7. Kim, J.Y., E.K. Kim, and S.S. Kim, Micro-nano hierarchical superhydrophobic electrospray-synthesized silica layers. J Colloid Interface Sci, 2013. 392: p. 376-381.

8. Kim, E.K., C.S. Lee, and S.S. Kim, Superhydrophobicity of electrospray-synthesized fluorinated silica layers. J Colloid Interface Sci, 2012. 368(1): p. 599-602.

9. Brown, N.A., et al., Electrospray deposit structure of nanoparticle suspensions. Journal of Electrostatics, 2017. 90: p. 67-73.

10. Stankus, J.J., et al., Fabrication of cell microintegrated blood vessel constructs through electrohydrodynamic atomization. Biomaterials, 2007. 28(17): p. 2738-2746.

11. Jaworek, A. and A.T. Sobczyk, Electrospraying route to nanotechnology: An overview. Journal of Electrostatics, 2008. 66(34): p. 197-219. 
12. Wang, Y., et al., Electrospray cone-jet breakup and droplet production for electrolyte solutions. 2012. 99(6): p. 64003.

13. Gañán-Calvo, A.M., J. Dávila, and A. Barrero, Current and droplet size in the electrospraying of liquids. Scaling laws. Journal of Aerosol Science, 1997. 28(2): p. 249-275.

14. Jaworek, A., Electrospray droplet sources for thin film deposition. Journal of Materials Science, 2007. 42(1): p. 266-297.

15. Rietveld, I.B., et al., Morphology control of poly (vinylidene fluoride) thin film made with electrospray. 2006. 298(2): p. 639-651.

16. Lei, L., et al., Obtaining Thickness-Limited Electrospray Deposition for 3D Coating. ACS Appl Mater Interfaces, 2018. 10(13): p. 11175-11188.

17. Bodnár, E. and J. Rosell-Llompart, Growth dynamics of granular films produced by electrospray. Journal of Colloid and Interface Science, 2013. 407(0): p. 536-545.

18. Altmann, K., et al., Electrospray ionization for deposition of ultra-thin polymer layers - principle, electrophoretic effect and applications. Journal of Adhesion Science and Technology, 2013. 27(9): p. 988-1005.

19. Banerjee, S. and M.K. Mazumder. Adhesion of charged powders on metal surface in powder coating process. in Conference Record of the 1993 IEEE Industry Applications Conference Twenty-Eighth IAS Annual Meeting. 1993.

20. Prasad, L.K., J.W. McGinity, and R.O. Williams, Electrostatic powder coating: Principles and pharmaceutical applications. International Journal of Pharmaceutics, 2016. 505(1): p. 289-302.

21. Bailey, A.G., The science and technology of electrostatic powder spraying, transport and coating1This invited paper is in commemoration of Prof. Bill Bright who contributed so much to electrostatics and especially to powder coating both directly and by stimulating numerous researchers in this field.1. Journal of Electrostatics, 1998. 45(2): p. 85-120.

22. Kim, J.W., et al., Direct and dry micro-patterning of nanoparticles by electrospray deposition through a micro-stencil mask. Journal of Micromechanics and Microengineering, 2009. 19(2): p. 025021.

23. Yan, W.-C., J. Xie, and C.-H. Wang, Electrical Field Guided Electrospray Deposition for Production of Gradient Particle Patterns. ACS Applied Materials \& Interfaces, 2018. 10(22): p. 18499-18506. 24. Lee, S., H. Koo, and S. Cho, Mask-less patterning of organic light emitting diodes using electrospray and selective biasing on pixel electrodes. Applied Physics Letters, 2015. 106(17): p. 173303.

25. Al-Milaji, K.N. and H. Zhao, Fabrication of superoleophobic surfaces by mask-assisted electrospray. Applied Surface Science, 2017. 396: p. 955-964.

26. Mayr, M.B. and S.A. Barringer, Corona Compared with Triboelectric Charging for Electrostatic Powder Coating. Journal of Food Science, 2006. 71(4): p. E171-E177.

27. Li, Q., et al., A systematic investigation into a novel method for preparing carbon fibre-carbon nanotube hybrid structures. Composites Part A: Applied Science and Manufacturing, 2016. 90: p. 174-185.

28. Kim, J.W., et al., Direct and dry micro-patterning of nanoparticles by electrospray deposition through a micro-stencil mask. Journal of Micromechanics and Microengineering, 2009. 19(2).

29. Liontas, R. and J.R. Greer, 3D nano-architected metallic glass: Size effect suppresses catastrophic failure. Acta Materialia, 2017. 133: p. 393-407.

30. Meza, L.R., S. Das, and J.R. Greer, Strong, lightweight, and recoverable three-dimensional ceramic nanolattices. Science, 2014. 345(6202): p. 1322-1326.

31. Schaedler, T.A., et al., Ultralight Metallic Microlattices. Science, 2011. 334(6058): p. 962-965.

32. Han, D., et al., Micro 3D Printing of a Temperature-Responsive Hydrogel Using Projection Micro-Stereolithography. Scientific Reports, 2018. 8(1): p. 1963.

33. Han, D., et al., Soft robotic manipulation and locomotion with a $3 d$ printed electroactive hydrogel. ACS applied materials \& interfaces, 2018. 10(21): p. 17512-17518.

34. Yang, C., et al., 4D printing reconfigurable, deployable and mechanically tunable metamaterials. Materials Horizons, 2019.

35. Sun, C., et al., Projection micro-stereolithography using digital micro-mirror dynamic mask. Sensors and Actuators A: Physical, 2005. 121(1): p. 113-120.

36. Zheng, X., et al., Design and optimization of a light-emitting diode projection micro-stereolithography three-dimensional manufacturing system. Review of Scientific Instruments, 2012. 83(12): p. 125001.

37. Han, D., et al., Rapid multi-material 3D printing with projection micro-stereolithography using dynamic fluidic control. Additive Manufacturing, 2019. 27: p. 606-615. 